\title{
From opacimetry to nanoparticle characterization: regulation, research and new national standards for exhaust gas analyzers in Europe
}

\author{
P. Ulbig* \\ Physikalisch-Technische Bundesanstalt (PTB), Bundesallee 100, 38116 Braunschweig, Germany
}

Received: 15 December 2009 / Accepted: 5 February 2010

\begin{abstract}
The measurement of the car exhausts plays an important role in many countries all over the world. Often, national legislation lays down limits to preserve the environment and, in addition, special regulations exist for the metrological control of exhaust gas analyzers. In the 90s, opacimetry was introduced as a fast and simple measuring technique to qualify the exhaust systems of cars equipped with Diesel engines. However, for modern cars equipped with soot reduction systems, conventional opacimetry reaches its limits. Therefore, new exhaust gas analyzers enter the market and, in parallel, the regulations in many countries will be modified. In terms of traceability, corresponding national standards must be developed for setting up a traceability chain for this new kind of instrument. For this reason, research is carried out with the aim of implementing particle counter standards for aerosols. In some countries, new set-ups are under construction which may lead to future intercomparisons. Today thousands of opacimeters are still in use, yet new instruments should comply with some of the given requirements in order to allow a smooth transition from one technique to the other. In other words, new instruments should combine the principles of two different "worlds": opacimetry and particle counting. The purpose of this paper is to give an overview of the development of new exhaust gas analyzers as well as of new national standards and new regulations in this field.
\end{abstract}

Keywords: Opacimetry; exhaust gas analyzers; regulations; nanoparticle characterization

\section{Introduction}

The number of cars worldwide is continuously increasing. Whereas about 500 million cars are on the road today, in the year 2030 there will be about 1.4 to 2.3 billion cars [1]. The exhaust from these cars is a reason for regulating car emissions in many countries. One has to distinguish between the regulations concerning the type approval of new cars and engines, respectively, and also the type approval of exhaust gas analyzers, especially for periodical control of cars in use.

For cars equipped with a diesel engine, usually the particulate mass emitted in $\mathrm{g} / \mathrm{km}$ for a defined driving cycle is taken as a measure for type approval of new passenger car engines. For this quantity, limit values given by the national regulations of an assortment of countries are shown in Figure 1 [2]. This example shows that there is a wide variety of different requirements all over the world. However, most countries are interested in reducing the current limit values for particulate mass in the near future, so that the detection limit for the particulate mass determined by gravimetry will be reached in some countries. Therefore, a corresponding change has been carried out in Europe. Regulation EC/692/2008 [3] takes into account

^ Correspondence: Peter.Ulbig@ptb.de

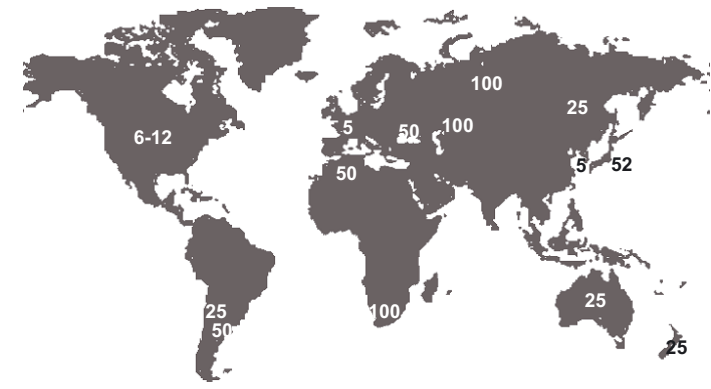

Fig. 1. Current regulations on particulate mass emissions of passenger cars with a diesel engine for an assortment of countries in $\mathrm{g} / \mathrm{km}[2]$.

the particle number for the first time. A limit value of $6 \times 10^{11}$ particles $/ \mathrm{km}$ for so-called "dry particles" is valid. A special procedure for the thermal treatment of the exhaust is given to obtain particles which are not covered by volatiles.

As a consequence, the relevant European authorities or technical departments carrying out tests need calibrated particle counting instruments which give comparable results. This is a very important requirement to avoid giving disadvantages to competing car manufacturers. For this reason, national standards are necessary which allow for 
the setting up of a traceability chain. It must be emphasized that particle counting instruments usually measure a particle number concentration (number of particles per volume). As tests for new engines are carried out for a defined driving cycle, there has to be a recalculation of the measured results to finally obtain the number of particles per kilometer.

Besides the type approval of new engines, many countries have regulations concerning the periodical exhaust emission testing of passenger cars and trucks. This started in approx. 1990 concerning the exhaust of diesel engines. An easy-to-use measuring technique for this purpose is the so-called "opacimetry". It represents an optical principle where the transmission of light of a given wavelength through a heated measuring cell is measured. Usually, part of the exhaust gas is pumped through this cell and absorbs some of the light. The requirements on this kind of instrument are given by the joint document OIML R-99/ISO 3930 [4].

Due to the decreasing limit values for particulate mass in exhaust gas, the sensitivity of the opacimeters has now reached its limit. Especially when cars are equipped with soot reduction systems, the detection limit of opacimeters is reached. For this reason, there are several options for national legislative bodies regarding how to proceed. The easiest option is to stop the regulation on this topic, due to the argument that the reduction of emissions has been so large, that the engines now emit a sufficiently clean exhaust. However, it is known that soot reduction systems are subject to thermal stress and to the load of already collected soot. Therefore as a second option, the pressure difference between the inlet and outlet of a diesel particulate filter could be a measure. If this pressure difference is too low, it is an indication of a malfunction due to either larger channels being formed in the filter or even a broken filter. Usually a differential pressure sensor is used which gives a signal to the on-board diagnostic (OBD) system. In Germany it has been required since 2006, that this OBD signal has to be scanned for periodical exhaust gas testing. However, if a particulate filter is distorted very slowly over time it may be useful to measure the exhaust of a diesel engine periodically, but this time with a suitable particle measuring instrument, which replaces an opacimeter. This third option may also be very useful as nanoparticles seem to have harmful properties especially concerning health, e.g. triggering lung cancer.

\section{Primary particle counter standards}

The basis for a traceability chain concerning particle counting is a national primary standard for particle counting. This standard has to combine four different steps as shown in Figure 2.

The first step is to provide defined particles. For this step it has to be decided whether monodisperse or polydisperse particles should be taken and which diameter the particles should have. As a starting point usually monodisperse certified particles are taken e.g. latex particles with a geometric diameter of 100 or $200 \mathrm{~nm}$. However, besides

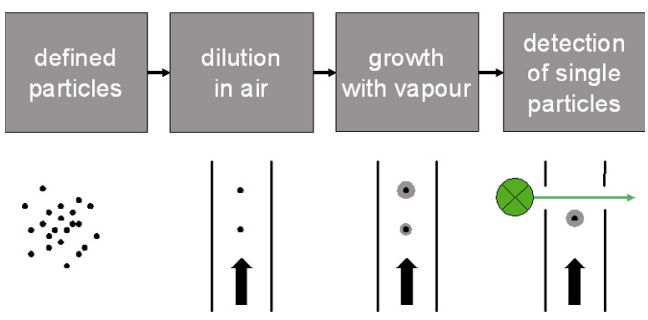

Fig. 2. The four basic steps of a primary particle counter standard.

the number concentration of particles, the size distribution may be of interest, too. For this purpose, a particle generator is necessary which provides a stable particle size distribution for a certain range, e.g. from 10 to $1000 \mathrm{~nm}$. In any case the particles must be provided in a gas stream. If the particles are provided in a liquid phase, then this suspension has to be transferred into the gaseous state before proceeding.

The second step consists in diluting the particles in a gas stream. The aim is to isolate the particles from each other to achieve the precondition for counting them individually. Usually very large dilution ratios must be performed. Depending on the initial concentration, dilution ratios of e.g. 1:100000 may be necessary. For this reason, the traceable and very precise measurement of the volume flow plays an important role.

In a third step the particles flow through a defined vapour, which usually consists of water or butanol. In this step the particles increase their diameter due to vapour condensation on the surface of the particles. This step is necessary, especially for the smaller particles with a diameter below approx. $300 \mathrm{~nm}$, because in the last step the particles are led through a laser beam for the counting process. Usually wavelengths between approx. 400 and $600 \mathrm{~nm}$ are used. In combination with a suitable detection system each single particle can be detected and counted when it passes through the laser beam.

When such a process has been implemented, the aim is to calibrate commercial particle counting instruments by comparison. Therefore the total gas flow with particles has to be divided into equal partial flows. However, as there is uncertainty concerning each step of the whole process, one has to be aware of setting consistent conditions for this comparison. For example it should be mentioned that some particles are in contact with the wall of the tube. This contact has to be minimized to keep the particles in the gas stream and to ensure that the same number of particles reach the particle counter and the equipment under test, too.

From a metrological point of view it is interesting to compare this kind of counting procedure with a metrologically independent one (Fig. 3). For this reason an electrometer can be used as an alternative.

In this case the particles must be charged and detected by the electrometer. One must make sure that each particle will have the same charge, e.g. one negative charge. This is necessary to be able to rely on the measured current. Each charge must correspond to one particle. For 


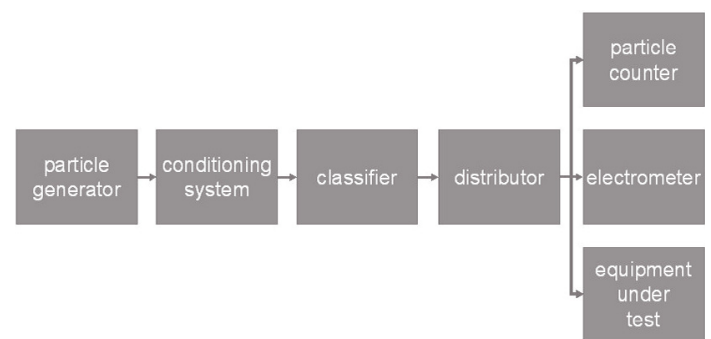

Fig. 3. Block diagram of a primary particle counter standard used for calibration.

this technique, it is a challenge to measure the extremely small current which can be found in the range of a few femtoamperes, with a sufficiently low uncertainty.

In principle, a primary particle counter standard which is also able to measure the particle size distribution consists of several parts: a particle generator to provide a stationary particle stream, a conditioning system to adjust conditions like temperature or humidity, a classifier to adjust the particle size range and finally a distributor to produce equal partial particle streams for each kind of measuring system.

Currently there is only one national particle counter standard worldwide in Switzerland (METAS, Bern) $[5,6]$. It was designed for the calibration of commercial particle counting systems in terms of particle number and particle size distribution. However, research to investigate many important aspects of such standards is being carried out within the EURAMET Joint Research Project "Traceable characterization of nanoparticles" within the framework of the EC ERA-NET Plus programme (iMERA plus) from June 2008 to May 2011. In this project a consortium of eight National Metrology Institutes across Europe has been formed. The aim is to develop methods needed for a traceable measurement of (amongst others) number concentration, its size and the shape of nanoparticles in aerosols. Different particle generators will be explored to generate traceable nanoparticle streams for aerosol measurement systems. In addition research is carried out to investigate the process of charging nanoparticles with one charge and to measure the corresponding current. This project will help to build primary particle counter standards in a few European countries and to establish intercomparisons for obtaining a sound basis concerning traceability.

Primary particle counting standards for aerosols are important for different sectors. Besides the exhaust of cars, the traceable long-term measurement of nanoparticles in ambient air is under discussion for future legislation. As more and more synthetic nanoparticles are produced and may enter the biosphere, a corresponding monitoring could be mandatory in the future. For this reason, national particle counting standards will play an important role in meteorology, toxicology, health and the protection of labour. As these standards can be extended relatively easily to measure particle size distribution, an additional benefit is generated.

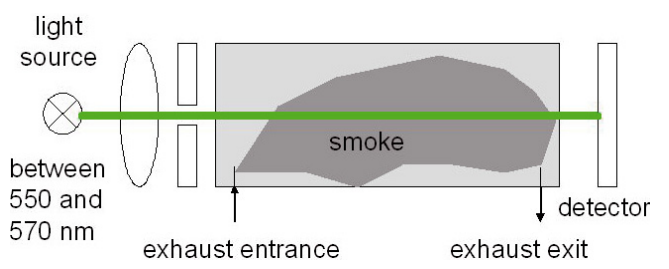

Fig. 4. Schematic diagram of an opacimeter according to ISO 11614.

\section{Periodical exhaust emission testing}

For the periodical exhaust emission testing, opacimeters are commonly used. Opacimeters are partial-flow systems, which measure online the exhaust gas opacity or the light absorption coefficient of exhaust gas from diesel engines. According to ISO 11614 [7] they consist of a light source with a spectral peak wavelength between 550 and $570 \mathrm{~nm}$ and a heated measuring cell to prevent the condensation of water vapour from the exhaust gas (Fig. 4).

Emission reducing systems like particulate filters lead to a remarkable reduction of particles. On the one hand the total number of particles is reduced by about two orders of magnitude (e.g. from $10^{8}$ particles per $\mathrm{cm}^{3}$ to $10^{5}$ or $10^{6}$ particles per $\mathrm{cm}^{3}$ ). On the other hand particulate filters cut off particles with a mobility diameter greater than approx. $250 \mathrm{~nm}$. This significant change in the composition of the exhaust gas leads to a zero signal of common opacimeters. The absorption of light by the nanoparticles is so low that usually the sensitivity of the opacimeters is too low to give a signal. What happens if a particulate filter changes its structure due to the thermal stress? Theoretically, there are two options: if a sufficient number of particles with a diameter larger than approx. $250 \mathrm{~nm}$ is able to pass through the filter, then the opacimeter will give a certain signal. If only particles with a diameter lower than $250 \mathrm{~nm}$ pass, but to a higher extent, then an opacimeter may not give a signal in any case. To be sure whether a particulate filter still works correctly or not, a better measuring technique with a higher sensitivity has to be used. Since conventional particle counting systems are certain to measure low concentrations of nanoparticles, but are too expensive for use in the periodical surveillance of cars, new kinds of measuring instruments with less expensive are necessary. Nowadays passenger cars which show extremely different exhaust behaviour from very low to very high particulate emissions can be found on the road. Modern instruments therefore, should allow the measurement of the common range of opacimeters and relatively low concentrations of nanoparticles, too.

Which measuring principles exist in general to measure particle number concentration down to the nanometer range? In fact there are three different measuring principles:

- High voltage charging.

- Photo acoustic spectroscopy.

- Laser light scattering. 
High voltage charging consists of two electrodes: a high voltage electrode within the exhaust gas stream to charge particles and a low voltage working electrode, where the particles are deposited. Since each particle should have one charge, the corresponding current is a measure for the particle number.

Photo acoustic spectroscopy usually works with laser light directed through an exhaust stream. The light hitting a particle leads to an increase in the temperature of the particle and the surrounding air. A local pressure change occurs. Under normal conditions these pressure differences will immediately disappear. If, however, the laser light is not applied with constant intensity but as a wave of changing intensity, then the result will be a pressure wave, in other words an acoustic signal which can be detected with a microphone.

If laser light scattering is applied, then usually light with a very short wavelength (e.g. about 200 to $600 \mathrm{~nm}$ ) is used to cause interaction between the light and the particles. By detecting the scattered light, a measure for the particle concentration can be obtained. However, the smallest diameter of a detectable particle is directly related to half of the laser wavelength, which limits this technique to a particle diameter of about $100 \mathrm{~nm}$ at present.

It has to be mentioned that all three techniques give only a particle number concentration in the ideal case of equally sized particles (i.e. monodisperse particles). But in reality exhaust gas consists of polydisperse particles with a corresponding particle size distribution. For this reason, the signals of the three measuring principles are more or less related to the masses of the particles. Therefore, the instruments indicate usually a mass concentration of the particle stream with the unit $\mu \mathrm{g} / \mathrm{m}^{3}$. As all three principles could be very sensitive, the detection limit could fall to about $5 \mu \mathrm{g} / \mathrm{m}^{3}$. At the other end of the measuring range typically about 500 to $900 \mathrm{mg} / \mathrm{m}^{3}$ can be detected easily, so that the conventional measuring range of an opacimeter is covered in principle. However, correlations must be developed giving a link between mass concentration and opacity. Fortunately, the first results in this direction show a sufficient accordance.

Due to the indication of a mass concentration the question arises: how these instruments can be linked to a primary particle counter standard in terms of traceability? This question is really a challenge and currently a topic of research, but it can be answered in general as follows: a primary particle counter standard finally gives an indication of a particle number concentration. This information is not sufficient to calculate a mass concentration. Only if, in addition, a particle size distribution can be obtained from the primary standard and some information concerning the density and the shape of the particles is available, a mass concentration can be calculated. In terms of traceability and especially uncertainty investigations are necessary to prove whether the common limits in legal metrology concerning the maximum permissible errors can be reached for the equipment under test. Future research will show whether this challenging goal can be achieved.

\section{Conclusions}

The change from opacimetry to nanoparticle characterization in order to extend measuring capabilities to lower concentrations and smaller particles leads to the development of primary particle counter standards. These standards must give information about particle size distribution too. Only in this case cheap measuring techniques like high voltage charging, photo acoustic spectroscopy or laser light scattering will get traceability at a national standard and may be used in legal metrology. More research is necessary to establish such national standards, but as mentioned previously, there are also numerous additional benefits for other fields of interest. As more and more nanoparticles will be produced e.g. for use in medicine or consumer products, suitable measuring techniques for counting and sizing nanoparticles in aerosols are of high interest.

Acknowledgements. The author wishes to thank Dr. Jürg Schlatter (METAS, Bern) for many fruitful discussions. Thanks are also addressed to the colleagues at PTB concerned with the interdisciplinary Working Committee on particle measuring techniques.

\section{References}

1. Umwelt- und Prognose Institut e.V., Folgen einer globalen Massenmotorisierung, UPI-Report No. 35, 1995

2. DELPHI, Worldwide Emissions Standards - Passenger Cars \& Light Duty Trucks, DELPHI annual report, 2009

3. EC/692/2008, Commission Regulation (EC) No. 692/2008 of 18 July 2008 implementing and amending, Regulation (EC) No. 715/2007 of the European Parliament and of the Council on type-approval of motor vehicles with respect to emissions from light passenger and commercial vehicles (Euro 5 and Euro 6) and on access to vehicle repair and maintenance information, 2008

4. Instruments for measuring vehicle exhaust emissions. Part 1: Metrological and technical requirements. Part 2: Metrological controls and performance tests, OIML R-99, 2008

5. J. Schlatter, Measurement of Particles in Aerosols Challenges and Solutions, Chimia 63, 665 (2009)

6. J. Schlatter, LAPAZ - a laser particle counter as a primary number concentration standard, J. Aerosol Science 35, 901 (2004)

7. ISO 11614, Reciprocating internal combustion compressionignition engines - Apparatus for measurement of the opacity and for determination of the light absorption coefficient of exhaust gas, 1999 